\title{
Design, assessment and evaluation of structural stabilization system for weather buoys using a moving foil
}

\author{
Mina Malek Azari ${ }^{*}$, Jose Victorio Salazar Luces and Yasuhisa Hirata
}

\begin{abstract}
This paper proposes a novel, simple and high-efficient submerged structural stabilization system for a self-stabilizing buoy that provides a stable platform for oceanographic and meteorological sensors. The proposed stabilization system is a Seesaw-like Tuned Mass Damper (STMD) combined with a foil and is designed in a way to harvest the activation energy from incoming waves. A fixed rudder in the rear underside of the buoy, as well as physical properties of the buoy, help to keep its balance in yaw and roll directions while encountering lateral waves. Therefore, the orientational stabilization of the whole structure is achieved by controlling only the pitch deviation. The proposed model is then simulated in a marine dynamic analysis software. Two different shapes of a thin rod and a foil were proposed for the sliding mass (slider) of the STMD and their performances are investigated and compared to each other in two different modes of passive and active control. In the passive mode, different mass ratios were conducted for the thin rod-shaped slider (i.e., a ratio of the effective mass of the stabilizing system to that of the whole structure) and a mass ratio of $8 \%$ could suppress the pitch deviation up to $81.3 \%$ in the resonance condition. The same STMD system with a mass ratio of $2 \%$ suppresses the pitch deviation by only $7 \%$. In the active mode, the STMD with a thin rod-shaped slider and a mass ratio of $8 \%$ exhibits only $49.1 \%$ suppression effect in a multiwave condition. However, by changing the shape of the slider to a foil while controlling its angle of attack (AOA) mechanically, the system with the mass ratios of $2 \%$ and $8 \%$ could suppress the pitch deviation of the buoy up to $69.4 \%$ and $79.1 \%$, respectively.
\end{abstract}

Keywords: Passive control, Active control, Seesaw tuned mass damper, Posture stabilization, Offshore structure

\section{Introduction}

Forecasting and predicting natural hazards has been a continuing concern. Correct and real-time data collection and transmission is one of the applicable methods to answer this issue. In this regard, the stabilization of meteorological and oceanographic sensors mounted on floating weather buoys is of great importance [1,2]. It is preferred to have a reliable and low-cost stabilization system that is easy to deploy and would not require frequent maintenance [3].

Wind sensors such as Light Detection and Ranging (LiDAR) module mounted on weather buoys play a key

\footnotetext{
*Correspondence: m.malek.azari@srd.mech.tohoku.ac.jp Department of Robotics, Graduate Faculty of Engineering, Tohoku University, Aoba, Sendai, Japan
}

role in obtaining reliable measurements of the wind profile and surface wave properties $[4,5]$. The most practical and commercialized stabilizers used for this purpose are the gyroscopic and gimbal types, which are large, heavy and costly and require frequent maintenance $[6$, 7]. Another popular solution is using parallel hydraulic arms to actively control the posture of a plate on which the module is mounted. Studies have shown that this mechanism is unable to respond to large deflections and suffers from the same shortcomings of gyroscopic stabilizers [8]. Likewise, vision systems are attached to the buoys to monitor unattended maritime areas. These systems require further stabilization to prevent the image sequence from being disturbed by irregular vibrations $[3$, $9,10]$. Although there are some mechanical systems proposed for stabilization of vision systems [11], the most 
common stabilization approach is to use a motion compensation software to apply some algorithms and image stabilization frameworks to the collected data [12]. Even though these approaches resolve the mechanical stabilization drawbacks, they are computationally demanding and their application is limited to a small range of vibrations.

The majority of the offshore structures such as wind turbines, jacket platforms, risers, and spar structures include structural stabilization systems to compensate for the orientation deviation of the whole structure against the external disturbances. General approaches proposed for this purpose are categorized as passive, active, and semi-active ones [13]. Usually, the structural stabilization systems are either mounted on or embedded inside the main structure. This type of Tuned Mass Dampers (TMD) are designed to mechanically damp the vibration either passively, actively or even semi-actively [14-17]. The efficiency of these systems is associated with their mass, stiffness and damping coefficient. Antirolling tank devices and Tuned Liquid Column Damper (TLCD) systems are also structural stabilization systems that are commonly used in boats and wind turbines [18, 19]. These stabilizers consist of a U-shaped container partially filled with a viscous liquid that is free to oscillate as a mass of damper. Although these stabilizers are popular and commonly used, they still require a large space inside the structure. In the meantime, there are few cases, in which the frame of the structural stabilization system undergoes a seesaw-like excitement, so-called Seesaw Active Mass Damper (SAMD). A similar approach has been applied to vibration suppression of tall buildings subjected to large seismic excitations [20]. Nevertheless, due to limited available space in weather buoys and their nonlinear response against external disturbances, far too little attention has been paid to the stabilization of the whole buoy. On the other hand, the unique properties of the foil-shaped blades moving through a fluid (i.e., lift and drag effects) have been always known as an alternative source of energy. The well known fin stabilizers and wave devouring propulsion systems (WDPS) are successful examples of this type that are used for roll stabilization of the moving ships and propulsion of boats, respectively [21-23].

In this research, a novel and simple system is designed to stabilize the orientation of the buoy by utilizing ocean wave energy and applying submerged Seesaw-like Tuned Mass Damper (STMD). Two different shapes of a thin rod and a foil are proposed for the sliding mass of the system and their performances are investigated and compared to each other in two different control modes of passive and active. To the best of our knowledge, the previous works have only been focused on stabilizing each of the sensors mounted on the weather buoys separately, either mechanically or by using algorithms on the obtained data. These approaches are often costly, energy-consuming and complicated $[6,12]$. Moreover, in previous works the proposed TMD stabilization mechanisms are either mounted on or embedded inside the main structure and have no contact with the surrounding water [14-17]. The proposed system in this study, however, provides a stabilized buoy, as a result of which, the sensors mounted on it might no longer require individual mechanical stabilization systems. Moreover, in the active mode control, only one actuator is required to stabilize the whole buoy structure carrying several sensors. Additionally, the proposed comparison between different shapes of sliders in the proposed mechanism may inspire future works using a combination of structural stabilization systems and a foil-shaped mass in various areas. The outline of this paper is as follows: first, the final concept and working principles of the proposed system will be described. Second, the dynamic model and controlling strategies will be disclosed. In the final section, the simulation results and discussion will be provided.

\section{Concept and working principles}

The final conceptual scheme of the stabilized buoy is illustrated in Fig. 1. The structure is formed of three main parts: a typical buoy with a fixed rudder at its rear underside, wave energy harvesting floaters connected to the four corners of the buoy, and a submerged stabilization mechanism.

\section{Buoy with a fixed rudder}

Inspired by the boat-shaped buoys such as a 6-meter NOMAD buoy [24], which is well-known for its highly directional and quick rotational response, a rudder is fixed to the rear underside of the buoy. In the proposed structure, the buoy tends to always be heading the incoming waves. Following any change in the direction of the incoming waves, the fixed rudder causes a rotation of the buoy around its central axis to turn it towards the incoming waves. This is due to the difference between the effective area against incoming waves (see Fig. 1).

\section{Wave energy harvesting floaters}

Using wave-induced forces to suppress the external disturbances is the main motivation for this paper. Studies have shown that a one-directional mechanism could be more efficient than multi-directional mechanisms in harvesting energy from ocean waves [25]. Among all the proposed methods in the literature, mechanisms that can use the wave energy in one of the heave, pitch or surge directions, are popular [25]. The shape and size of a floater are of the key elements to pick the right motion mode. Based 


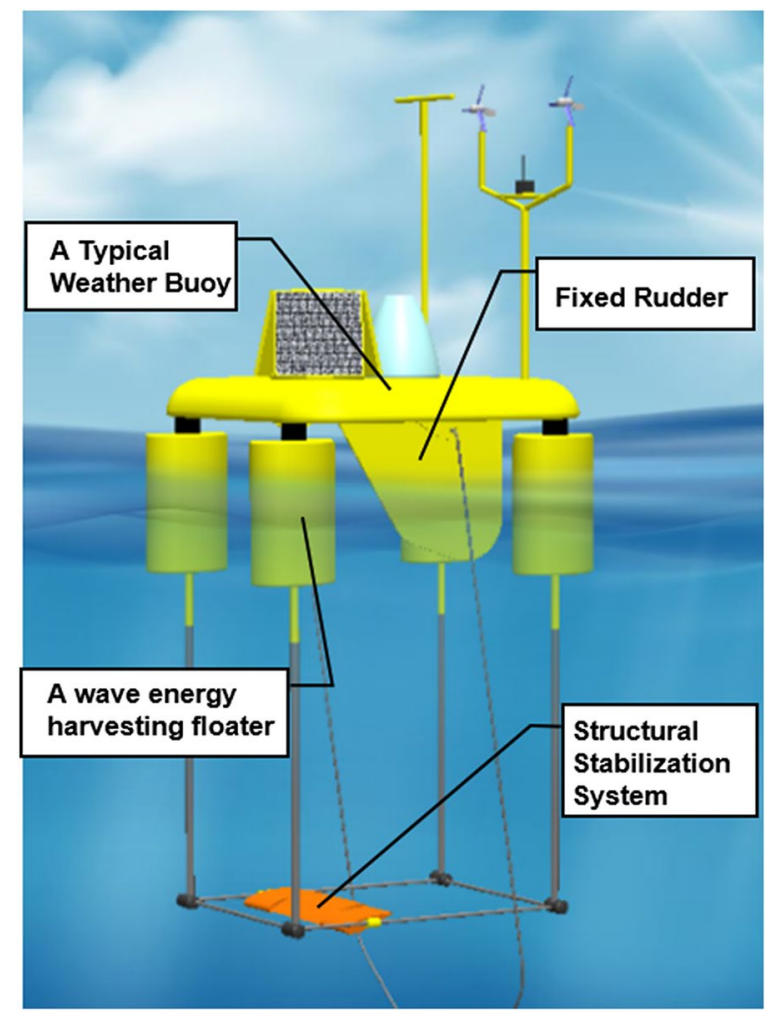

Fig. 1 Concept of final stabilized buoy. Three main parts of proposed stabilized buoy structure, a typical weather buoy with a fixed rudder, four wave energy harvesting floaters at each corner of the buoy, and the structural stabilization system are shown in the figure

on the definitions provided elsewhere [26], if the effective length of a floater is less than $1 / 6$ of the wavelength, it is considered as a small body, i.e., the heave motion is the most volumetric efficient and the other modes of motion could be neglected. As shown in Fig. 1 one cylindrical floater is placed at each of the four corners of the buoy. Therefore, when a wave reaches the buoy, the floaters at both ends will follow the crest and trough of the incoming wave. This will result in a harmonic heave and pitch motion in the floaters and the buoy, respectively. This way the floaters will excite the submerged stabilization system while preventing the buoy from rolling over against lateral waves.

A typical floating buoy has 6 degrees of freedom (DOF). Mooring a buoy to the sea bed will keep its position in an acceptable range reducing its DOF to only pitch, roll, and yaw rotations. As a result of a balanced model and attaching floaters at the four corners of the buoy, its roll rotation could be neglected. In addition, the fixed rudder would cause a constraint in yaw rotation. Consequently, the stabilization of the buoy in only pitch direction could possibly result in an acceptable stabilization of the buoy platform.

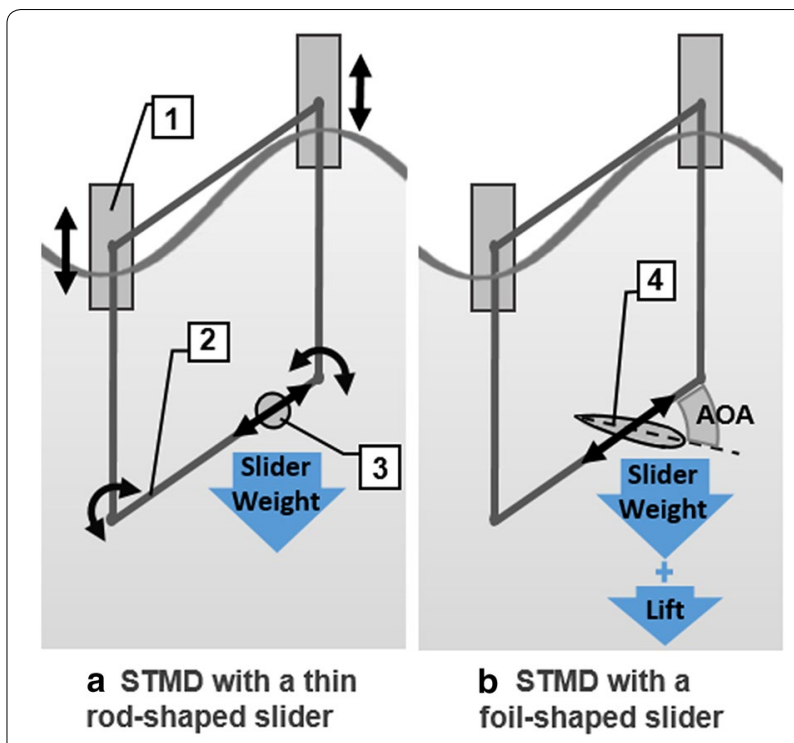

Fig. 2 Mechanism of the proposed structure. (a.1) Surface waves cause a heave motion in floaters, (a.2) The underwater frame swings due to the parallel mechanism, (a.3) The slider moves along the frame with a phase difference and its weight results a suppression force, (b.4) Changing the shape of the slider to a foil, rises lift force as an additional suppression force

\section{Submerged stabilization mechanism}

In order to meet the main goal of this research, i.e., controlling the pitch motion of the buoy via a simple and reliable system without need for any external actuators, a submerged STMD connected to the heaving floaters is proposed. The main strategy of STMD is similar to the widely applied TMD structural stabilization systems. The incoming waves result in a harmonic heave motion in each floater due to the pressure difference between the top and bottom surfaces of the floater. As shown in Fig. 2, this motion induces a pitch rotation in the buoy as well as the frame of the STMD due to the parallel design of the whole structure. A sliding mass (slider) is mounted on the submerged STMD frame and is free to move along it. The potential energy of the slider causes a linear motion along the submerged frame, in response to its oscillation. Eventually, the phase difference between the STMD frame's rotation and the slider's linear motion suppresses the pitch deviation of the buoy. Besides, unlike previous studies in TMD systems, there is direct contact between the slider and the surrounding water and thus its shape is one of the determinative factors alongside its mass in the proposed STMD's performance. For this aim, changing the shape of the slider from a thin rod to a foil, as seen in Fig. 2, leads to an additional resistive force arising from its motion. The hydrodynamic forces acting on a foil could be divided into two main forces of drag and lift, which are related to its velocity. As it is well-known in the 
hydrodynamics, the drag force is a resistive force in the direction of a foil's velocity. On the other hand, the lift force is a result of a non-zero angle of attack (AOA) of the foil and pushes the foil in a direction perpendicular to its forward velocity direction, i.e., up and down (see Fig. 2). By adjusting the AOA of the foil, the magnitude and the direction of the lift force could be controlled. Therefore, the lift force is treated as an external source of force to be used in the suppression of the buoy's pitch deviation.

\section{External forces}

In order to suppress the pitch deviation of the buoy by a structural stabilization system, it is required to understand the fundamentals of the active forces on the whole structure connecting the buoy to the submerged STMD. As explained at the end of the "Wave energy harvesting floaters" section, the buoy is assumed to have only a pitch motion while the floaters at its corners show a harmonic heave motion. Fig. 3 shows a heaving floater that faces linear waves. Based on the definitions provided elsewhere $[25,26]$, the water surface elevation in the floater's location (see Fig. 3) is calculated as

$$
\xi(t)=\frac{H}{2} \cos (k x-\omega t)
$$

where $H, k, \omega$, and $x$ are the wave height (i.e., crest to trough), the wave number, the wave frequency, and the

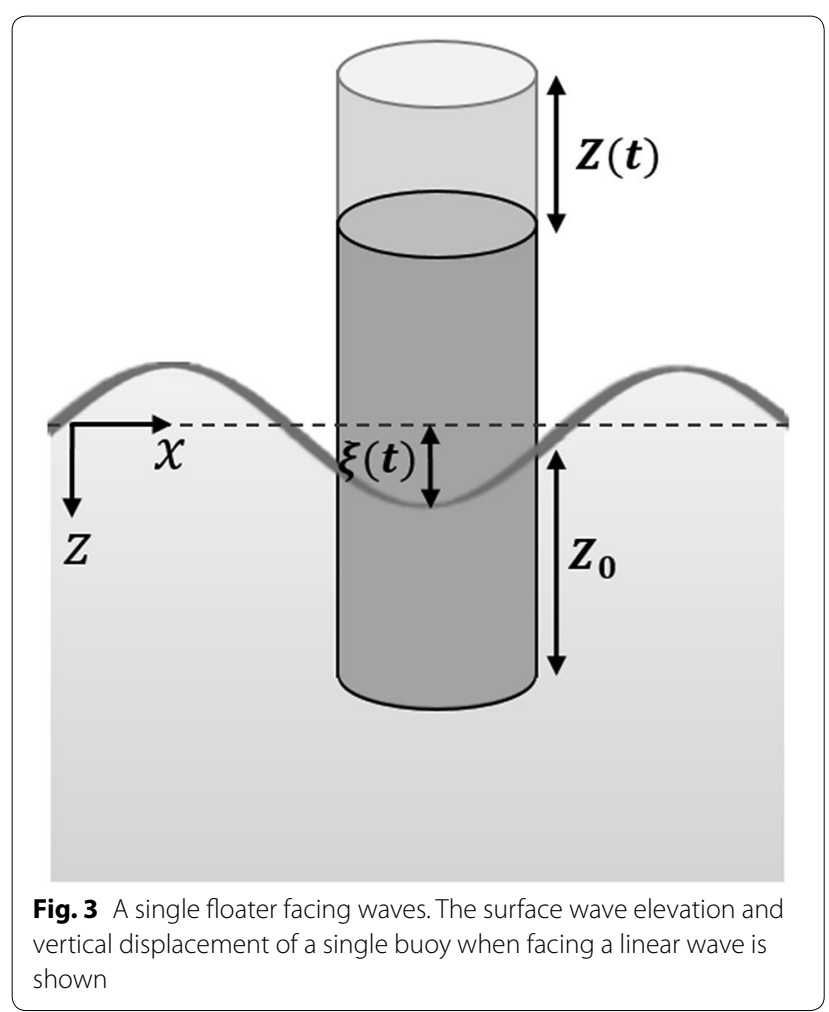

horizontal position of the floater with respect to the origin at the mean water level, respectively. Here, the $x$ is a constant value because the floater has only a heave motion and its horizontal location is assumed to be fixed. The water surface elevation is a result of the elliptical motion of the water particles. The vertical and horizontal motion of the water particles in the floater's location (with respect to the origin) could be written as follows:

$$
\begin{aligned}
& \xi_{Z}(t)=\frac{H}{2} \cos (k x-\omega t) \frac{\sinh (k Z(t)+k d)}{\sinh (k d)} \\
& \xi_{x}(t)=\frac{-H}{2} \sin (k x-\omega t) \frac{\cosh (k Z(t)+k d)}{\sinh (k d)}
\end{aligned}
$$

where $d$ is the water depth and the $Z(t)$ is a vertical displacement of the floater, which does not necessarily follow the water surface elevation $\xi(t)$ (see Fig. 3). Note that the $Z_{0}$ in Fig. 3 is the immersed height of the floater at its stationary condition [27]. The velocity and acceleration equations of the water particles will be obtained from first and second derivatives of the equations (2) and (3). However, since by getting to deep waters (where the water depth $d$ exceeds half of a wave length [25]), the motion of the water particles transfers to a circular one (not elliptical), the terms related to water depth will be simplified and removed from the derived velocity and acceleration equations according to the dispersion relation [25]. Therefore, the vertical velocity and acceleration of water particles in the floater's location are obtained as follows:

$$
\begin{aligned}
& \dot{\xi}_{Z}(t)=\frac{g k H}{2 \omega} e^{k Z(t)} \cos (k x-\omega t) \\
& \ddot{\xi}_{Z}(t)=\frac{g k H}{2} e^{k Z(t)} \cos (k x-\omega t)
\end{aligned}
$$

where $g$ is the gravity acceleration.

In general, each floater experiences four groups of forces, described as follows:

$$
\begin{aligned}
& F_{b}=\rho_{w} g A\left[\xi_{Z}(t)-Z(t)\right] \\
& F_{a . m}=C_{a} \rho_{w} A\left[Z_{0}+\xi_{Z}(t)-Z(t)\right]\left[\ddot{Z}(t)-\ddot{\xi}_{Z}(t)\right] \\
& F_{F . K}=\rho_{w} A\left[Z_{0}+\xi_{Z}(t)-Z(t)\right] \ddot{\xi}_{Z}(t) \\
& F_{d}=C_{d} \dot{Z}(t)
\end{aligned}
$$

where $F_{b}$ is the difference between gravity and buoyancy force and is proportional to the height of the displaced water. $F_{a . m}$ and $F_{d}$ are the added mass force (proportional 
to body acceleration) and damping resistance force (proportional to body velocity), respectively, being raised due to the floater's motion. $F_{F . K}$ denotes the excitation force, also known as the Froude-Krylov force. The excitation force is proportional to wave acceleration and is felt by the floater when it is kept fixed against incoming waves. $\left[Z_{0}+\xi_{Z}(t)-Z(t)\right]$ is the submerged height of the floater (see Fig. 3) with respect to the wave surface. The $\left[\ddot{Z}(t)-\ddot{\xi}_{Z}(t)\right]$ is the relative acceleration between a floater and the water particles. $\rho_{w}, C_{d}, C_{a}$, and $A$ are water density, damping resistance coefficient, added mass coefficient, and effective area of a floater, respectively.

Looking closer at the equations above, all the active forces are functions of either displacement, velocity or acceleration. Thus the environmental condition of the buoy with floaters could be replaced by a set of a mass-springdamper system at each floater. Fig. 4 displays a planar schematic of the buoy platform and the floaters at both ends being connected to a set of spring and damper indicating the external active forces.

Likewise, a moving body inside the water experiences the Froude-Krylov force and added mass force as comes below:

$$
\begin{aligned}
& F_{F . K . s}=\rho_{w} V_{s} \ddot{\xi}_{r}(t) \\
& F_{a . m . s}=C_{a . s} \rho_{w} V_{s}\left[\ddot{\xi}_{r}(t)-\ddot{r}(t)\right]
\end{aligned}
$$

where

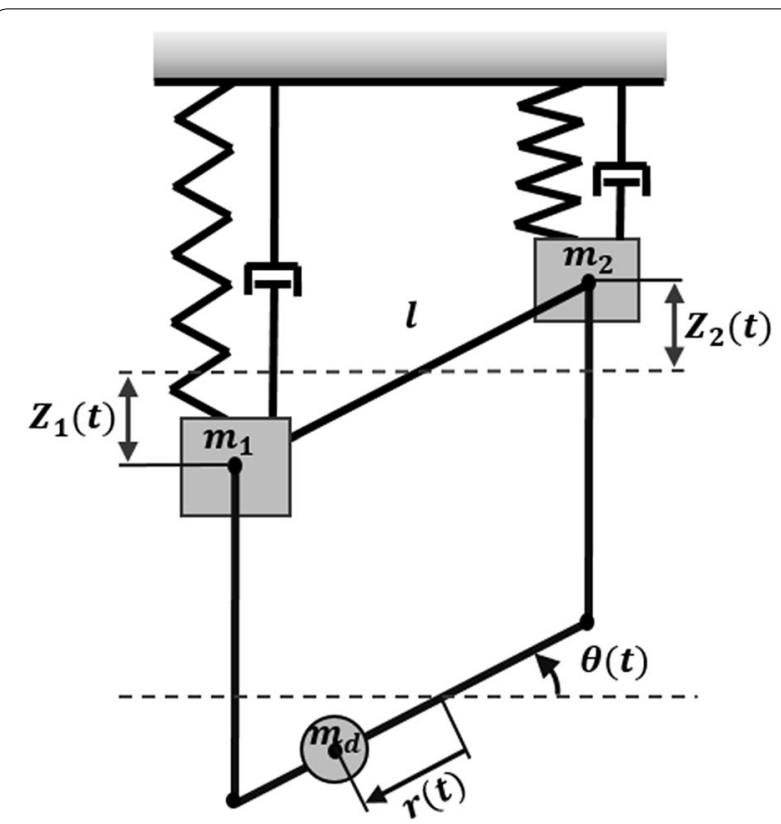

Fig. 4 Floaters attached to a spring and a damper. A set of mass-spring-damper system is replaced with the environmental active forces

$$
\ddot{\xi}_{r}(t)=\frac{g k H}{2} e^{k Z_{s}} \cos [k r(t)-\omega t]
$$

here, $C_{a . s}, V_{s}$, and $Z_{s}$ are added mass coefficient of the slider, the volume of the slider, and height of the slider from mean water level respectively. $Z_{s}$ is assumed to be constant due to small-angle approximations at pitch deviations (i.e., deviation is less than $10^{\circ}$ ). $r(t)$ and $\ddot{r}(t)$ are the linear position of the slider on the STMD frame and linear acceleration of the slider alongside the STMD frame respectively, as shown in Fig. 4. It is important to note that in case of the foil-shaped slider lift and drag forces arise (see Fig. 2) that have a substantial role in the dynamics of the model while in the equations above, we have only focused on a thin rod-shaped slider, which experiences neither drag nor a lift force due to its small cross-section area. Therefore, the resistive drag force and the lift forces have been neglected while calculating the effective forces. It is worth mentioning that considering the fact that the wave-induced forces are strongest on the surface, the stabilization mechanism is connected to the floaters using vertical rods and deployed in a depth of more than half of a wavelength, where the wave-induced forces are less than $4 \%$ of those in the surface.

Demonstrating the effect of a foil-shaped slider on the STMD's performance in the suppression of the buoy's pitch deviation is the primary objective of this research. Despite the fact that a dynamic model of the system is essential for designing a controller, as long as our focus is the study of the motion of bodies without regard to the controlling forces, a kinematic model is sufficient to control a simulated system. The proposed mechanism consists of a buoy with floaters and submerged STMD is a parallel mechanism (see Fig. 2). Taking into account that obtaining a kinematic model for parallel mechanisms in coupled systems is demanding, an alternative modeling approach is considered. System identification methods are widely used in controlling the offshore structures [28, 29]. In this approach using a limited number of measurements of the inputs and outputs, a model is predicted for the system. In this regard, first, a specific range of signals are input to the system. Second, the resulting output signals are recorded. Based on the recorded inputs and outputs, an identification algorithm is used to determine the optimal parameters that govern the kinematic behavior of the system [29]. Specifications of the input signal have a crucial role in the accuracy and validity of the identified model. It is important to ensure that the input data used to determine the model covers all the range of frequencies and all amplitudes that are likely to be encountered during system operation. In order to benefit from the system identification approach, a fundamental step is the design of the experiment, either by a physical or a 
simulated model, to examine the inputs and outputs. In this work, a simulated model has been provided, which will be described in the "Simulation and results" section. Note that the system identification methods are only applicable to the thin rod-shaped slider due to the existence of an extra parameter (i.e., AOA of the foil) and technical limitations of the simulation in the foil-shaped slider.

\section{Control strategies}

The controlling approaches studied in this research are based on a combination of the STMD concept and the foil effect. First, the control of the thin rod-shaped slider in the passive mode (see Fig. $5 \mathrm{a}$ ) is explained. Then to confirm the efficiency of the STMD combined with a foil, the active mode control is studied in both thin rod and foil-shaped sliders, as schematically shown in Fig. 5b, c, respectively.

\section{Passive control}

In this approach, due to the passive nature of the control, no actuator is used to move the slider of the STMD. As an alternative, the mass and stiffness of the thin rod-shaped slider in STMD are tuned according to the natural frequency of the buoy and its operational condition. Therefore when encountering external waves, the STMD gets excited and utilizing the potential energy of the slider originated from its mass and stiffness, suppresses the pitch deviation of the buoy. The main point of
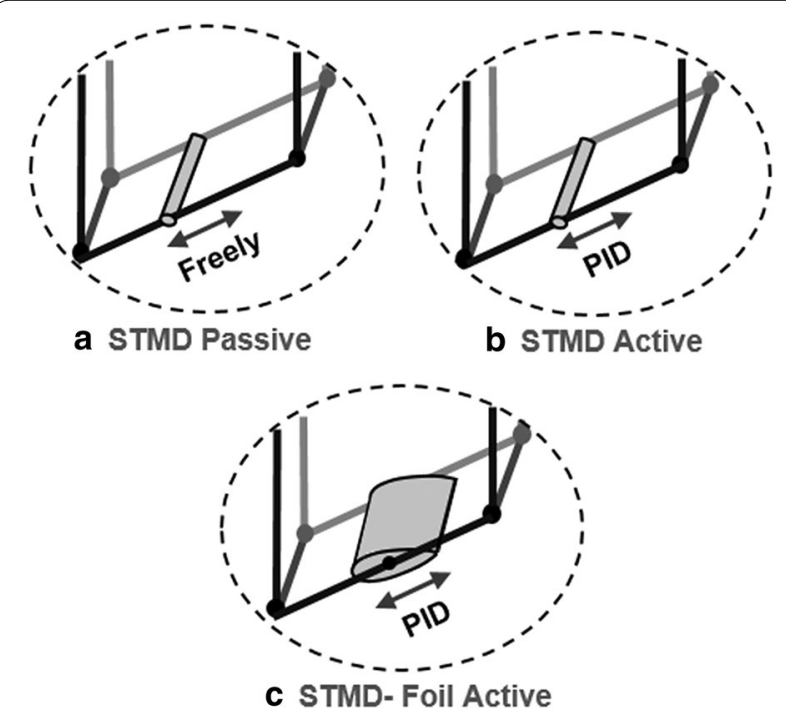

Fig. 5 Schematic concept of controlling strategies. a A thin rod-shaped slider moves along the submerged STMD's frame with no control. b A thin rod-shaped slider moves along the submerged STMD's frame with a PID controller. c A foil-shaped slider moves along the submerged STMD's frame with a PID controller this strategy is the absence of any external control force on the linear motion of the slider along the frame. The foil-shaped slider is not studied in passive mode control in the current paper because changing the shape of the slider from a thin rod to a foil will add extra hydrodynamic forces to the governing dynamics of the system including drag and lift coefficients, which depend on the AOA and physical properties of the foil (e.g., its profile and span length). Therefore, in addition to the mass ratio and stiffness of the slider, the AOA of the foil as well as its shape should be tuned to the optimum values. Tuning all these parameters requires solving several optimization problems, which falls out of the scope of this paper and are left for the future modifications. In this paper, we have considered STMD-Foil only in the active mode control.

\section{Active control}

The active mode control consists of two different cases; the thin rod and foil-shaped sliders. In both cases, an actively controlled linear motion is applied to the slider in order to suppress the wave-induced pitch deviation of the buoy. Note that in the case of the foil-shaped slider, no actuator is used for controlling its AOA. The AOA is designed to be controlled mechanically by a mechanism attached to the frame. Fig. 6 shows the motion of the foil-shaped slider along the frame. As seen, when the frame is subjected to a pitch deviation $(\theta(t))$, the active mode controller applies a linear motion $(r(t))$ to the

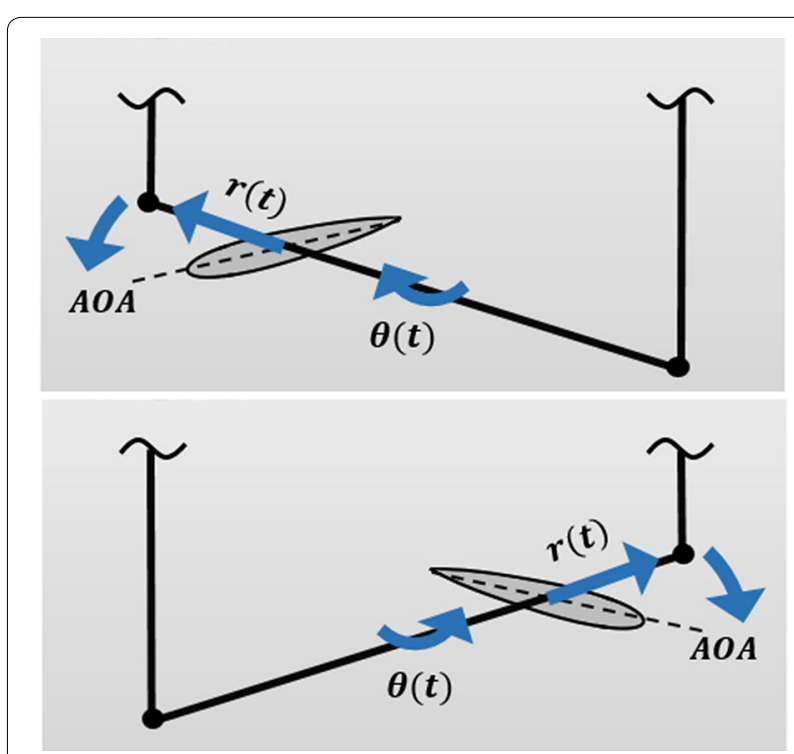

Fig. 6 Active control of foil-shaped slider. The figure shows the direction of the slider's linear motion $r(t)$ and its rotaion (AOA) with respect to the frame, in two cases of clockwise (upper figure) and counterclockwise (below figure) pitch deviation $\theta(t)$ of the frame 
foil-shaped slider to move it from the middle of the frame to the sides. However, the AOA of the foil-shaped slider is defined regardless of the controller's output and is calculated with respect to the frame and is set to increase from $0^{\circ}$ at the middle of the frame to a maximum angle of $3^{\circ}$ at the end points of the frame. The $3^{\circ}$ for the maximum AOA is selected based on the existing data set for the selected foil profile (NACA 0015) at low Reynolds numbers [30], based on which the drag coefficient $\left(C_{D}\right)$ decreases by increasing the AOA of the foil and meets a minimum value. Lower $C_{D}$ means lower actuation energy required for the linear motion of the slider. Moreover, by increasing the AOA of the foil-shaped slider with respect to the frame from $0^{\circ}$ to $3^{\circ}$, the ratio of the lift coefficient to drag coefficient (i.e. $C_{L} / C_{D}$ ) increases to almost 20 times. This leads to a larger lift force while keeping the drag force low. Note that for the simulations in this paper, the mechanically control of the AOA is applied by user defined functions that are independent from the active controller. In other words, in the active mode control, the motion of the slider at both thin rod and foil-shaped sliders is controlled by only one linear actuator along the STMD frame.

In the active control strategy, it is attempted to determine the linear position of the slider along the frame in such a way to damp out the pitch deviation of the buoy as efficiently as possible. A block diagram shown in Fig. 7 demonstrates an overall control flow of the system in the active mode control. Since it is desired to stabilize the buoy in a pitch direction, the desired input pitch angle of the control system is $0^{\circ}$. As shown in Fig. 7 , the controller receives the error in the pitch angle, which is exactly the pitch deviation of the buoy, and then applies the proper linear displacement of the slider in order to compensate the pitch deviation. Meanwhile, the controlling algorithm should be selected carefully to ensure that the obtained results in both thin rod and foil-shaped sliders are comparable. For this purpose, a proportional-integral-derivative

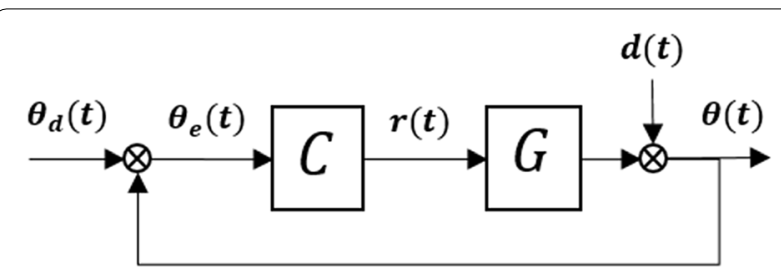

Fig. 7 Active control block diagram. The pitch stabilization control flow is shown in this figure. The control plant $G$ consists of the whole structure of buoy together with stabilization mechanism. $\theta$ and $\theta_{d}$ are the observed and the desired pitch angle (i.e., zero degree) of the buoy, respectively. The controller $C$ gets the pitch deviation $\theta_{e}$ and sends the proper linear displacement $r$ of the slider to the $G$. Wave induced external forces are input as a distubance $d$ to the system
(PID) controller is proposed. The fundamental challenge in designing a satisfactory PID controller is tuning the PID parameters. In this research, a MATLAB toolbox named Control System Toolbox (CST) is used for tuning the PID parameters of the active controller for a thin rodshaped slider based on the identified model from System Identification Toolbox of MATLAB. After utilizing different apps and algorithms provided in the CST, the best achievable results for the tuning were obtained by the manual tuning of the gains using the PID Tuner interface. Due to the absence of a model for the stabilization system with a foil-shaped slider, the CST could not be utilized for tuning the PID gains of the active controller in this case. Therefore, the application of a non-rule based tuning (manual tuning) method was inevitable in a foilshaped slider. In order to achieve adequate results in both cases (i.e. a thin rod and a foil-shaped slider), the steps considered for the manual tuning were derived from the Ziegler-Nichols method.

\section{Simulation and results}

To determine optimal design parameters in the realistic operational conditions, the proposed model of the buoy and floaters with a stabilization system is simulated using ProteusDS (PDS). PDS is a marine dynamic analysis software being widely used in ocean industries to model and evaluate various systems that are exposed to wind, current, and waves by simulating actual operational conditions [31]. A number of studies have performed PDS and provided reliable results being comparable with realworld data [32, 33]. In this regard, first, the performance of the fixed rudder is investigated. Then, a kinematic model is identified, i.e., a linear equation that relates the position of the thin rod-shaped slider with the pitch angle of the buoy. Finally, the passive control strategy and the active control strategy (PID controller) are implemented using an Application Programming Interface (API).

\section{Simulated model}

The physical parameters of the simplified simulated model in the PDS environment are listed in Table 1. Fig. 8 shows the simulated model in the PDS environment that

Table 1 Physical properties of simulated model

\begin{tabular}{llc}
\hline Parameter & Value & Unit \\
\hline Area of the buoy's platform & $6 \times 6$ & $\mathrm{~m}^{2}$ \\
Overall height of the structure & 15 & $\mathrm{~m}$ \\
Deployement weight & 6000 & $\mathrm{~kg}$ \\
Water density & 1025 & $\frac{\mathrm{kg}}{\mathrm{m}^{3}}$ \\
Water depth & 150 & $\mathrm{~m}$ \\
Wave type & Airy & \\
\hline
\end{tabular}




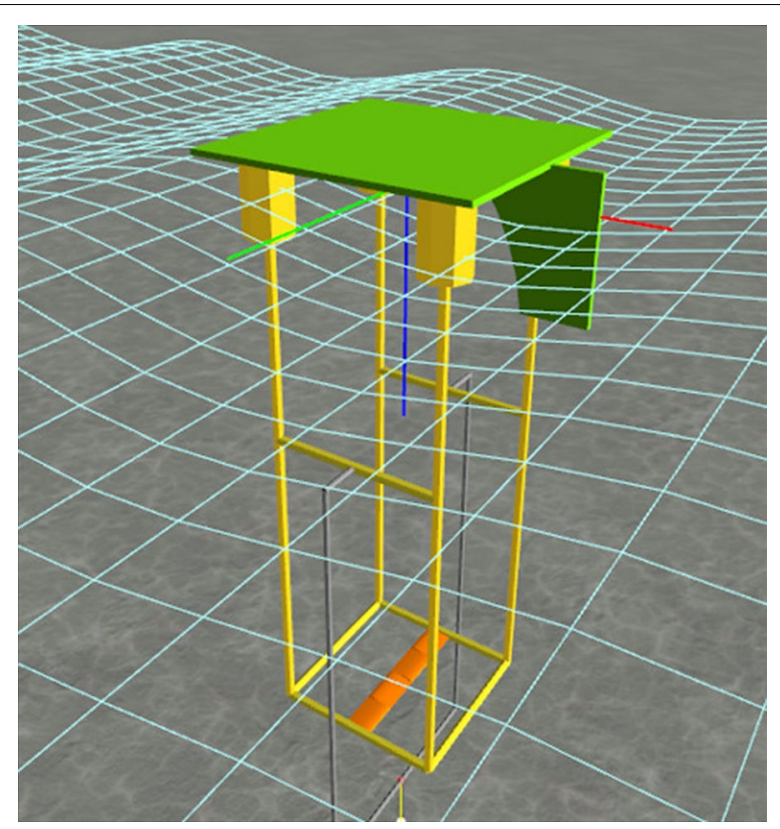

Fig. 8 Simulated model in the PDS environment. A typical buoy with a fixed rudder attached to the four floaters at corners and the submerged stabilization system is shown

consists of a buoy held above the sea level by four floaters at the corners. The STMD stabilization mechanism is connected to the floaters using rigid rods located deep enough to minimize the effect of the surface waves. The submerged STMD is in direct contact with surrounding water and consists of a frame on which a thin rod or foilshaped mass is sliding freely. A mooring cable connects the buoy structure's center of gravity to the seabed. The materials considered in the simulated model are selected based on a real model of buoy structure to ensure its applicability. To reduce the computational time, the model is mostly formed of cylinders and rectangles without rounding off the corner edges.

\section{Fixed rudder's performance}

In order to investigate the performance of the fixed rudder mentioned earlier in the "Buoy with a fixed rudder" section, the whole simulated structure was set to encounter waves from a lateral direction as illustrated in Fig. 9. Since the perpendicular component of the wave-induced force acts on the fixed rudder, lateral waves with heading angles between 10 to $90^{\circ}$ (i.e., the least and the most effective angles, respectively) were selected and applied during the simulations. In order to prevent the simulated structure from being drifted by external waves, it was moored to the seabed using a cable connected at the height of its center of gravity. The height and period of the incoming waves as well as the speed of the current were selected based on a real operational condition, as listed in Table 2, and kept fixed at different headings. The yaw rotation of the buoy was recorded during each simulation for 100 seconds. As evidenced in Fig. 10, the fixed rudder applies a yaw moment to the buoy engendered by the lateral wave-induced force. The resulted moment then turns the buoy's fore towards the incoming waves in less than one minute.

\section{Buoy structure model identification}

Determining the frequency bandwidth of a system's response at its free vibration is a common preliminary practice [29]. This study focuses on suppressing the pitch deviation of the buoy. Inside the PDS environment, the simulated model is initially displaced from its equilibrium position in the calm waters, i.e., an initial pitch angle of $10^{\circ}$ is applied to the buoy. In the absence of any external waves, the structure freely oscillates back to its equilibrium position. The time-domain response of the structure is obtained via simulation for 100 seconds at a sampling rate of 0.05 seconds. The frequency-domain data is calculated from time-domain data using the Fast Fourier Transformation (FFT). The natural response of the buoy against an initial pitch deviation for both time

Table 2 Wave conditions used in investigatin the fixed rudder's performance

\begin{tabular}{lll}
\hline Parameter & Value & Unit \\
\hline Wave height & 1 & $\mathrm{~m}$ \\
Wave period & 5 & $\mathrm{~s}$ \\
Wave heading & 45 & $\mathrm{deg}$ \\
Current speed & 0.75 & $\frac{\mathrm{m}}{\mathrm{s}}$ \\
Current heading & 45 & deg \\
\hline
\end{tabular}

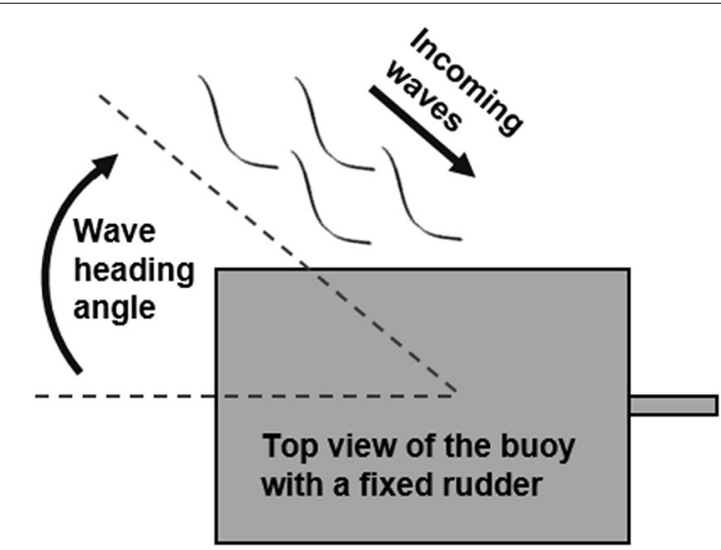

Fig. 9 Buoy with fixed rudder against lateral wave. A lateral wave with a heading of $45^{\circ}$ is reaching the buoy with a fixed rudder 


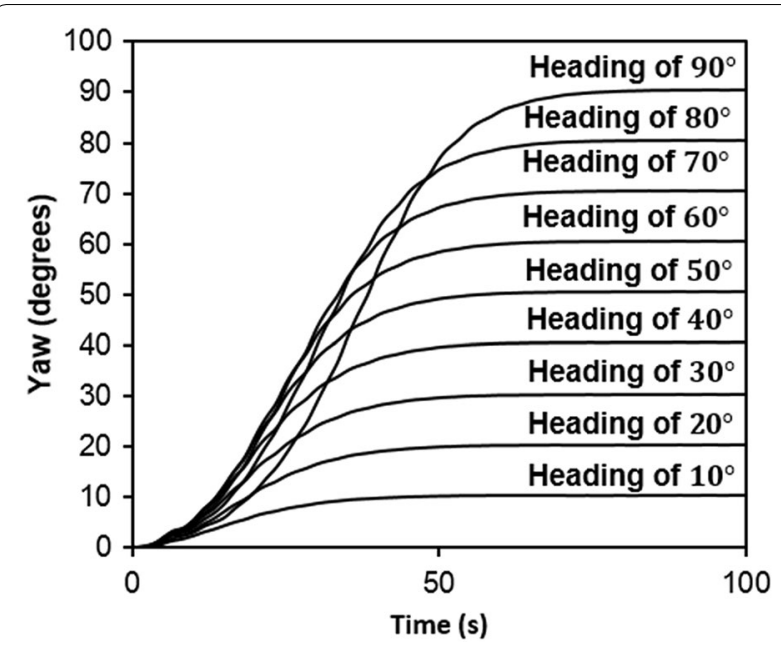

Fig. 10 Yaw response of the buoy to a lateral wave. Yaw rotation of the buoy with a fixed rudder against a lateral wave with a heading of $45^{\circ}$

and frequency domains is shown in Fig. 11. The obtained free response of the system indicates that the system experiences the resonance phenomenon in pitch rotation at the frequency of 0.24 Hertz (period of 4.16 seconds).
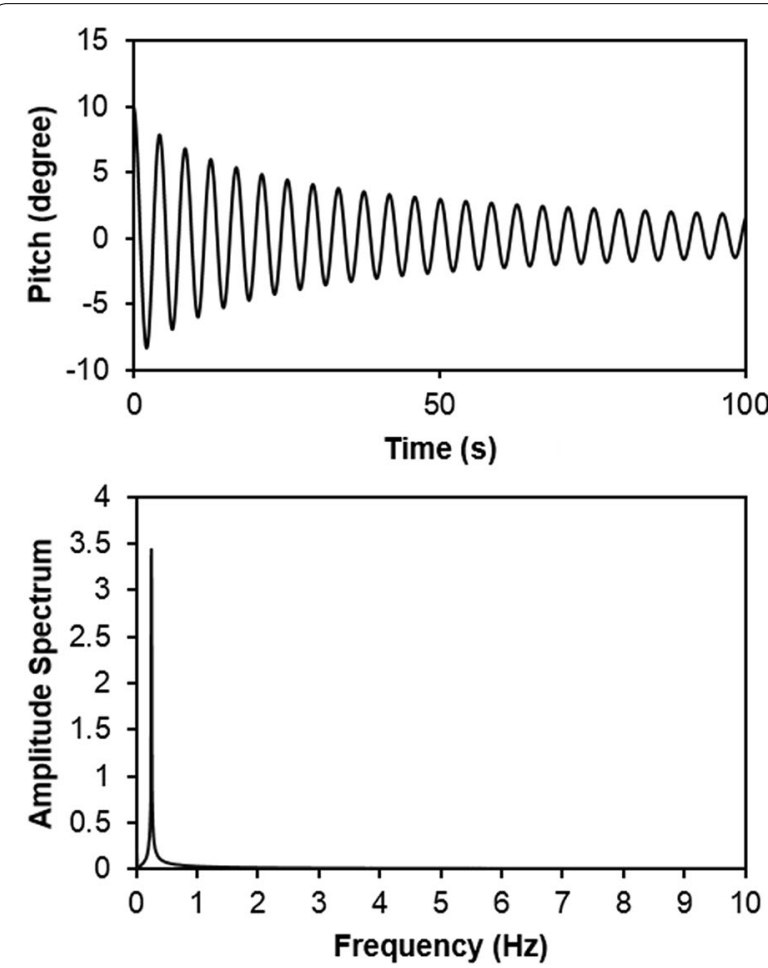

Fig. 11 Free vibration response of the system. Time-domain and frequency domain response of the buoy structure to an initial pitch displacement of $10^{\circ}$
Therefore, the input signal for identification should cover this frequency range.

In order to identify an applicable model, a multisine signal with random amplitude and period shown in Fig. 12 was input as a linear motion of the thin rodshaped slider along the STMD frame and the resulted pitch deviation of the buoy was recorded. Using MATLAB System Identification Toolbox, and the recorded input-output data (i.e., $r(t)$ and $\theta(t)$, respectively), the kinematic model which defines the pitch rotation of the buoy as a function of the linear motion of the thin rodshaped slider was identified. A transfer function of the system was obtained and used in tuning the parameters of the PID controller mentioned in the "Active seesawshaped tuned mass damper" section. Fig. 13 compares the response obtained by the best fit $(82.4 \%)$ model and the actual output from PDS.

\section{Control strategies implementation}

\section{Passive seesaw-shaped tuned mass damper}

In a passive control of the TMD systems, selecting a proper mass and stiffness for TMD is a crucial challenge. To avoid heavy loadings while achieving acceptable suppression rates, it is a common preference to assume a mass ratio (i.e., a ratio of the effective mass of the TMD to that of the whole structure) of $0.25-3 \%$ [14]. However, each structure has individual properties and requires proper tuning of the mass ratio. For this aim, a series of differing mass ratios in a range of $2 \%$ to $20 \%$ are considered. The resultant calculated mass of the slider is further added to its buoyancy weight. The stiffness of the slider is then calculated based on its mass ratio and the natural frequency obtained at "Identifying the buoy structure model" section.

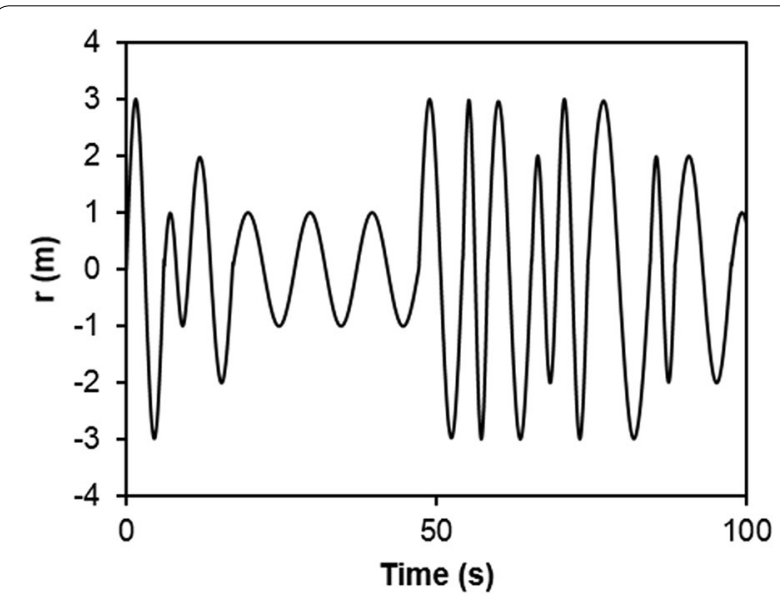

Fig. 12 The input multi-sine signal. Linear motion of the slider in STMD is input as a multi-sinusoidal signal for system identification 


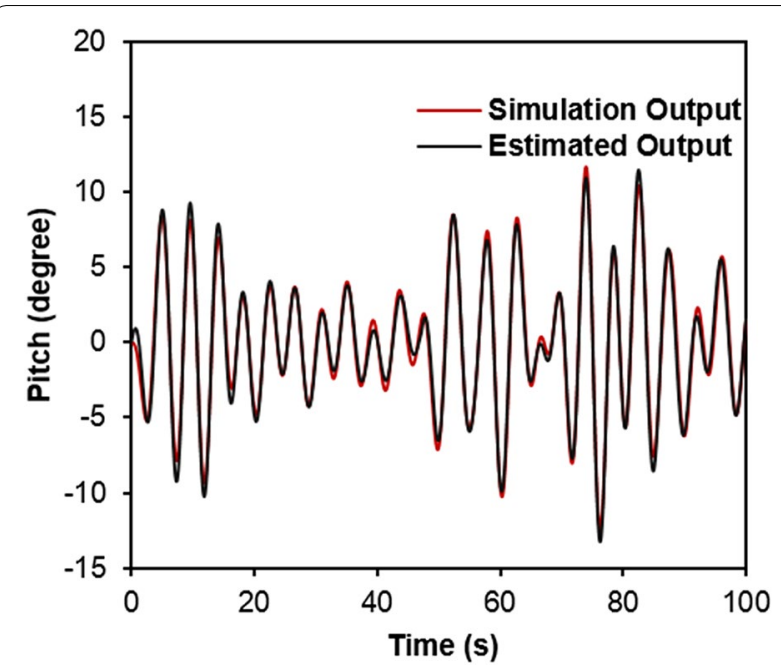

Fig. 13 Simulation output and estimated output of the system. Estimated output of the identified model fits the output of the simulation by $82.4 \%$ in response to a multi-sine input signal

To evaluate the performance of the STMD in a passive mode at each mass ratio ( $2 \%$ to $20 \%$ ), a resonance condition, as the most destructive condition, was considered in the simulation. For this purpose, the buoy structure model in PDS was simulated facing incoming waves with a frequency that equals the natural frequency of the buoy structure, i.e., $0.24 \mathrm{~Hz}$. Fig. 14 shows the frequency domain response of the buoy structure against a wave at a frequency of $0.24 \mathrm{~Hz}$, for the mass ratio of the $2 \%$ to $20 \%$. As expected, the STMD is able to suppress the pitch deviation of the buoy in a passive mode in a resonance condition. The rate of suppression increases as the mass ratio raises. However, a large weight may rise many difficulties in practice such as higher material cost, larger required transportation equipment and hardship of deployment and installation [34]. Therefore, a reasonable mass ratio should be selected considering both weight and suppression rate in each case. A standard deviation of the response at each case shown in Fig. 15 clearly demonstrates that in passive mode, increasing the mass ratio of the slider up to $8 \%$ rises the suppression rate of pitch deviation by $81.3 \%$ in resonance condition. Above $8 \%$, the suppression rate remains almost unchanged. Therefore, the $8 \%$ mass ratio for the slider is selected as a proper ratio in getting a reasonable vibration suppression in passive mode.

\section{Active seesaw-shaped tuned mass damper}

To evaluate the active mode control, a PID controller, as described in "Controlling strategies" section for both thin rod and foil-shaped sliders is tuned and the best achievable results are adopted. Controlling commands are implemented by $\mathrm{C}++$ programing language and transferred to PDS using its API.

During the simulation, a series of continuous linear waves with different periods and heights listed in Table 3 are considered. The values of periods and heights were selected based on the real wave properties recorded by weather buoys [35], including the resonance condition of the buoy structure. To evaluate the effectiveness of the proposed methods, six cases, either in passive or active mode, as listed in Table 4, were simulated. For each case, the controlling strategies were implemented and their time-domain response against different wave conditions (see Table 3) is shown in Fig. 16. To get a more clear understanding, a summary of the obtained results in various investigated conditions is provided in Fig. 18. As shown, the STMD with an $8 \%$ mass ratio in passive mode increases the overall pitch deviation of the buoy up to $11.9 \%$ against a series of continuous waves with different conditions. At the same environmental condition, in the active mode the overall pitch deviation of the buoy for the mass ratios of $2 \%$ and $8 \%$ [the least and most effective ratios in passive mode in resonance condition (see Fig. 15)], is suppressed up to $7.8 \%$ and $49.1 \%$, respectively. Finally, by changing the shape of the slider to a foil in the active mode, the pitch deviation of the buoy is suppressed by an overall $69.4 \%$ and $79.1 \%$ for the mass ratios of $2 \%$ and $8 \%$, respectively. Meanwhile, the linear motion of the slider along the frame in a time domain that causes the suppresion of buoy's pitch vibration is shown in Fig. 17. The data of the slider's linear motion were captured from the simulations conducted on the cases listed in Table 4 for the passive and active control.

\section{Discussion}

According to the information provided by the National Oceanic and Atmospheric Administration (NOAA) [36], wind-generated waves (known as wind waves) are counted as major waves. This is because of their relatively high amplitudes at lower periods compared to swell waves. Based on the real recorded data [35], the heading of the wind waves and the direction of the surface current remain almost unchanged during any given day. Therefore, the performance of the proposed fixed rudder in Fig. 10 is reasonably acceptable in a real operational condition. As shown in Fig. 10, the buoy rotates faster as the angle between wave heading and the longitudinal direction of the buoy increases. The larger angle, however, results in a larger yaw deviation of the buoy that should be compensated. It is worth mentioning that the performance of the buoy structure in the conducted simulations in Fig. 10 was tracked and no structural error was detected even after it was subjected to a wave with a heading angle of $90^{\circ}$. 



Fig. 14 Passive mode response of the buoy in frequency-domain. Buoy's pitch deviation with the STMD with mass ratios of $2 \%$ to $20 \%$ in a passive mode control is presented in frequency domain

Fig. 14 depicts the results obtained from a set of simulations that were run to understand the passive effects of mass ratio increment from 2 to $20 \%$ on the suppression rate of the pitch deviation in a resonance condition. During simulations, when there is no STMD mass on the buoy structure, the buoy structure resonates due to the equivalent magnitude of the wave's frequency and its natural frequency. As seen in Fig. 15, by adding a mass to the STMD's slider, the amplitude of the pitch deviation starts to decrease and drops significantly at a mass ratio of $5 \%$. From the mass ratio of $8 \%$ onwards, it remains relatively unchanged. Fig. 15 clearly shows that a mass ratio of $8 \%$ can reduce the pitch deviation of the buoy from around $12^{\circ}$ (at No STMD) to around $2^{\circ}$. Basically, in TMD stabilizers that are mounted inside or on top of the structures, the mass is connected to the main structure via a spring and a dashpot. The mass and spring are tuned based on a natural frequency of the main structure resulting in TMD's mass vibrating at a frequency equal to the natural frequency of the main structure. Then the damper dissipates energy from the whole system in the form of heat [14]. The proposed STMD in this paper, however, does not include any dashpot or a counterweight. Therefore, the only force that causes suppression on the pitch deviation of the buoy is the weight of the slider that produces a torque on the STMD's frame. For this reason, the mass ratio of $8 \%$ was selected as a proper ratio in getting a reasonable suppression in a resonance condition at a passive mode. In the meanwhile, the mass ratio of $2 \%$ was selected as the least effective ratio, since it reduces the pitch deviation of the buoy by the only $1^{\circ}$ (see Fig. 15).

Figure 16 clearly indicates that the resonation of the buoy can be suppressed effectively by using only the wave energy (i.e., without any external actuator or control on 


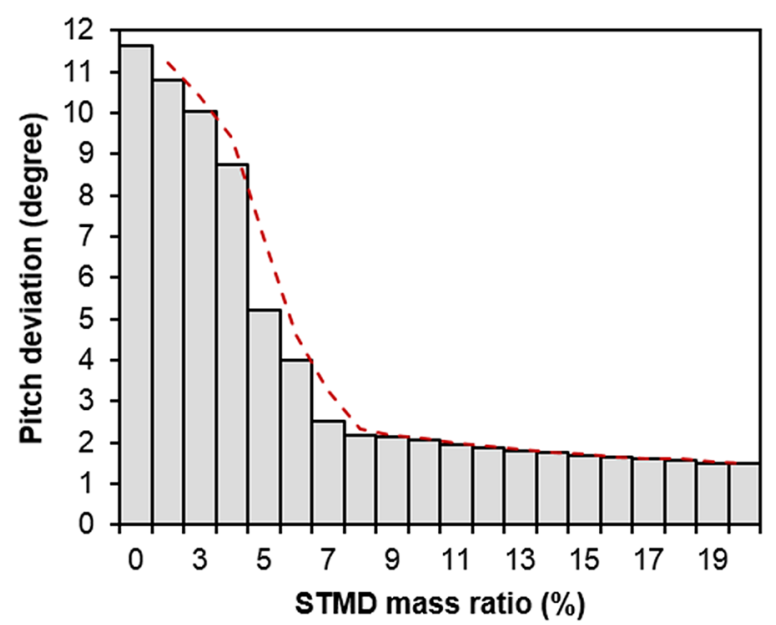

Fig. 15 Passive mode response of the buoy at different mass ratios of $2 \%$ to $20 \%$. A standard deviation of the buoy's pitch deviation with the STMD with mass ratios of $2 \%$ to $20 \%$ in a passive mode control is presented

Table 3 Random wave conditions for final evluation of proposed control strategies

\begin{tabular}{lll}
\hline Number of wave & Height $(\mathbf{m})$ & Period (s) \\
\hline 1 & 1 & 5 \\
2 & 1.5 & 8 \\
3 & 0.5 & 4.16 \\
4 & 0.7 & 10 \\
5 & 1.2 & 6 \\
6 & 0.5 & 8 \\
7 & 1 & 15 \\
8 & 0.5 & 3.5 \\
\hline
\end{tabular}

Table 4 Final simulated cases

\begin{tabular}{ll}
\hline Case name & Description \\
\hline No STMD & Buoy structure \\
STMD 8\% Passive & concept shown in Fig. 5a with a mass ratio of 8\% \\
STMD 2\% Active & concept shown in Fig. 5b with a mass ratio of 2\% \\
STMD 8\% Active & concept shown in Fig. 5b with a mass ratio of 8\% \\
STMD-Foil 2\% Active & concept shown in Fig. 5c with a mass ratio of 2\% \\
STMD-Foil 8\% Active & concept shown in Fig. 5c with a mass ratio of 8\% \\
\hline
\end{tabular}

the movement of the slider) in the STMD with a thin rod-shaped slider and a proper mass ratio of $8 \%$. However, not only it is unable to suppress the pitch deviation while encountering waves with different periods, it may further cause larger deviations. The main drawback of the passive mode control is that it is only applicable to a limited range of frequencies. In the active mode control, after carefully tuning the gains of the PID controller, the same thin rod-shaped slider with the same mass ratio (i.e., 8\%) is able to effectively reduce the pitch deviation of the buoy close to a half (see Fig. 18), while lowering the mass ratio to $2 \%$ results in much less suppression (only $7.8 \%$ ). This highlights the importance of the mass ratio in the STMD, even in the active mode. By taking this point into account and only changing the shape of the slider to a foil, the STMD becomes able to suppress the pitch deviation up to $69.4 \%$ and $79.1 \%$ for the mass ratios of $2 \%$ and $8 \%$, respectively. In other words, the suppression rate of the pitch deviation using the least efficient mass ratio of $2 \%$ increases by about nine times (8.89) by only changing the shape of the slider from a thin rod to a foil. However, in the case of $8 \%$ mass ratio, changing the shape of the slider from a thin rod to a foil increases the suppression rate by 1.6 times. This finding validates the effectiveness of the foil-shaped mass in the STMD performing inside the water in small mass ratios. However, the combination of two characteristics (i.e. a large weight and a foil-shaped) for a slider causes extra suppression force (lift force), which exceeds the required amount for the buoy's stabilization, and leads to a disturbance (rather than a suppression). Fig. 17 indicating the linear displacement of the slider along the frame is complementary to the results shown on Fig. 16. Comparison between the results of the active mode controls (either in the thin rodshaped or fin-shaped slider) in Figs. 16 and 17 reveals higher suppression rates for large displacements of the slider, which is reasonable since large displacement of the slider results in a larger moment on the frame. However, in the passive mode control, the sliders are not able to suppress the pitch vibration in some places even with a large displacement. A thin rod-shaped slider with an $8 \%$ mass ratio is able to suppress the pitch vibration even with the small range of displacement sicne its stiffness was initially tuned to the natural frequency of the system. This indicates that while the mass ratio of the slider has a significant role in the suppression rate, the stiffness of the STMD is also important in its performance.

\section{Conclusion}

In the present paper, the effect of a foil-shaped mass in increasing the efficiency of the STMD is investigated with the aim of stabilizing a weather buoy with mounted sensors on it. In the proposed structural stabilization system, a submerged STMD with a sliding mass is connected to a buoy. By mechanically harvesting energy from the incoming waves (i.e., passive mode control) that cause a pitch deviation on the buoy, the stabilization system gets excited and compensates for the pitch deviation up to $81.3 \%$ at a resonance condition. It is demonstrated that 


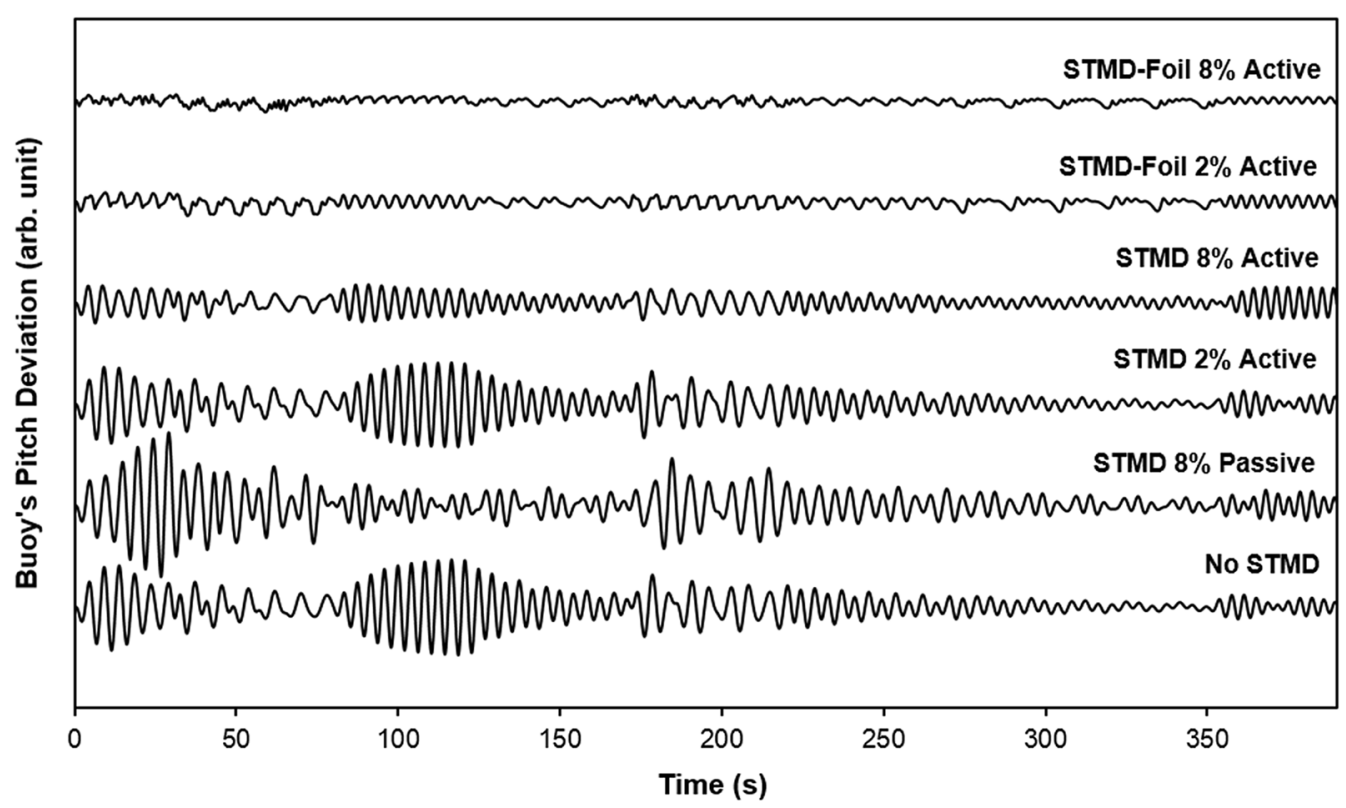

Fig. 16 Time-domain response of the buoy with different control strategies Buoy's pitch deviation with proposed control strategies in response to a series of continuous waves with different heights and periods is presented



Fig. 17 Time domain displacement of the slider at different control strategies The linear motion of the slider along the frame which causes the suppresion of the buoy's pitch deviation at different control strategies is presented

using a foil-shaped slider on the submerged STMD in the active mode control can effectively cause a suppression by $69.4 \%$ and $79.1 \%$ for various wave conditions including a resonance condition when the mass ratio of the slider is kept at $2 \%$ and $8 \%$ respectively. The obtained results from various simulations revealed that despite the proposed stabilization system shows remarkable performance in a passive mode, its performance is highly limited to a small frequency range of the incoming waves. To actively control the linear motion of the slider in a submerged STMD, 




Fig. 18 Response of the buoy with different control strategies A standard deviation of the buoy's pitch deviation with proposed control strategies in response to a series of continuous waves with different heights and periods is presented

one actuator is added to the STMD. As a result, the pitch deviation of the buoy is suppressed against waves with a wide range of frequencies and amplitudes. Furthermore, using a foil-shaped slider in the actively controlled STMD makes a significant difference in the effectiveness of the suppression rate. This verifies that the stabilization of the sensors on the weather buoys at various wave conditions could be possible with much lower costs by using a submerged STMD system combined with a foil. The obtained results can further be extended to a wide range of offshore structures that require stabilization systems such as spars, jacket platforms and especially wind turbines moored by tensioned cables in offshore places. As a future study, contributing authors intend to focus on developing a prototype and optimizing a semi-active suppression system applying a combination of STMD and foil approaches.

\section{Acknowledgements}

This research was partly carried out by marine dynamic analysis software, ProteusDSAPI. The authors wish to thank Dynamic System Analysis Ltd. group for their great support.

\section{Authors' contributions}

MMA proposed the stabilization mechanism, derived the theoretical solutions, identified the kinematic model of the proposed stabilization mechanism, carried out the simulations in PDS, proposed and implemented control strategies, assessed the results from simulations and drafted the manuscript. JVSL suggested the system identification approach, assisted the research and revised and refined the manuscript. YH led the research progress, assisted with the implementation of the control algorithm, secured funding for the PDS license and revised and refined the manuscript. All authors read and approved the final manuscript.

\section{Funding}

This work was not funded by any organization.

\section{Availability of data and materials}

Not applicable.

\section{Competing interests}

The authors declare that they have no competing interests.

Received: 22 March 2020 Accepted: 13 July 2020

Published online: 31 July 2020

\section{References}

1. Peng G, Bidlot J, Freitag HP, lii CJS (2014) Directional bias of tao daily buoy wind vectors in the central equatorial pacific ocean from november 2008 to january 2010. Data Sci J 13:79-87

2. Data Buoy Cooperation Panel: Developments in Buoy and Communications Technologies. USA (1996). Data Buoy Cooperation Panel

3. Fefilatyev S, Goldgof D, Lembke C (2009) Autonomous buoy platform for low-cost visual maritime surveillance: design and initial deployment. In: Hou, W. (ed.) Proceedings of SPIE-The international society for optical engineering. May 2009

4. Bischoff O, Yu W, Gottschall J, Cheng PW (2018) Validating a simulation environment for floating lidar systems. J Phys 1037:5

5. Antunano MAG (2019) Doppler with lidar systems data processing and application: An overveiw towards developing the new generation of wind remote-sensing sensors for off-shore wind farms. PhD thesis, Polytechnic University of Catalonia, Department of Signal Theory and Communication

6. Vepa KS, Duffey T, Paepegem WV (2016) Gyroscope-based floating lidar design proposal for getting stable offshore wind velocity profiles. Sea Technol 57(6):41-43

7. Schuon F, Gonzalez D, Rocadenbosch F, Bischoff O, Jane R (2012) Kic innoenergy project neptune: Development of a floating lidar buoy for wind, wave and current measurements. In: Proceedings DEWEK 2012: 11 th German Wind Energy Conference: Bremen, Germany: 7-8 November 2012. DSpace

8. Mes JC (2014) Stabilizing control system of a platform on a buoy for offshore wind assessment. Ph.D. thesis, Delft University of Technology, Department of Electric Engineering

9. Bakhoday-paskyabi M, Reuder J, Flugge M (2016) Automated measurements of whitecaps on the ocean surface from a buoy-mounted camera. Methods Oceanogr 17:14-31

10. Cai C, Weng X, Zhu Q (2018) Sea-skyline-based image stabilization of a buoy-mounted catadioptric omnidirectional vision system. EURASIP J 1:1

11. Grober D (2004) Stabilized camera and marker buoy for media coverage of aquatic events. US 6,718,130 B2

12. Tiana-Alsina J, Gutierrez MA, Wurth I, Puigdefabregas J, Rocadenbosch F (2015) Motion compensation study for a floating doppler wind lidar. International Geoscience and Remote Sensing Symposium (IGARSS) November, 5379-5382

13. Kandasamy R, Cui F, Townsend N, Foo CC, Guo J, Shenoi A, Xiong Y (2016) A review of vibration control methods for marine offshore structures. Ocean Eng 127:279-297

14. He EM, Hu YQ, Zhang Y (2017) Optimization design of tuned mass damper for vibration suppression of a barge-type offshore floating wind turbine. In: Hou, W. (ed.) Proceedings of the Institution of Mechanical Engineers Part M: Journal of Engineering for the Maritime Environment 2017, Vol 231 (1), pp. 302-315. Institution of mechanical engineers

15. Dinh VN, Basu B, Nagarajaiah S (2016) Semi-active control of vibrations of spar type floating offshore wind turbines. Smart Struct Syst 18:683-705

16. Chung LL, Lai Y, Yang CSW, Lien KH, Wu LY (2017) Semi-active tuned mass dampers with phase control. J Sound Vibr 332:3610-3625 
17. Babaei S, Amirabadi R, Taghikhany T (2017) Assessment of semi-active tuned mass damper application in suppressing seismic-induced vibration of an existing jacket platform. Int J Marit Technol 6:27

18. Ghaemmaghami A, Kianoush R, Yuan X (2012) Numerical modeling of dynamic behavior of annular tuned liquid dampers for applications in wind towers. Comput Aided Civil Infrastruct Eng 00:1-14

19. Tuan A, Shoji K, Minami K, Mita S (2008) Responses of roll damping on anti-rolling tank devices. OCEANS 2008-MTS/IEEE Kobe Techno-Ocean, $1-6$

20. Yoshida K, Fukui K, Watanabe T (1996) Active vibration control system for buildings subjected to horizontal and vertical large seismic excitation. In: Proceedings of the 36th Conference on Decision and Control Kobe, Japan. December 1996. IEEE

21. Qi Z, Jin H, Liu W, Xu Y (2014) Research on active fin stabilizer at low speed and its application to ship roll stabilization. OCEANS 2014-TAIPEI, $1-6$

22. Terao Y, Sakagami N (2015) Design and development of an autonomous wave-powered boat with a wave devouring propulsion system. Adv Robot 29:89-102

23. Beglerovic H, Hirata $Y$, Kosuge K (2017) Formation control of multiple passive type boats for sea surveillance. In: Proceedings of 2016 IEEE/SICE international symposium on system integration, Sapporo Convention Center, Sapporo, Japan, pp 869-874

24. 6-Meter NOMAD Buoy. https://axystechnologies.com/products/nomad -buoy/

25. Chakrabarti SK (2005) Handbook of offshore engineering, vol 2. Offshore structure analysis Inc, New York

26. Pecher A (2017) Handbook of Ocean Wave Energy. Springer, Berlin

27. Jahangir MH, Hosseini SS, Mehrpooya M (2018) A detailed theoretical modeling and parametric investigation of potential power in heaving buoys. Energy 154:201-209
28. Bacelli G, Coe RG, Patterson D, Wilson D (2017) System identification of a heaving point absorber: design of experiment and device modeling. Energies 10:24

29. Davidson J, Giorgi S, Ringwood JV (2016) Identification of wave energy device models from numerical wave tank data_-part 1: numerical wave tank identification tests. IEEE Trans Sust Energy 7:1-8

30. Airfoil Tools (NACA 0015 Airfoil). http://airfoiltools.com/airfoil/details?airfo il=naca0015-il

31. Dynamic System Analysis Ltd.: ProteusDS 2015 Manual. Victoria, BC, Canada (2015). Dynamic System Analysis Ltd

32. Beatty S, Roy A, Bubbar K, Ortiz J, Buckham B, Wild P, Steinke D, Nicoll R (2015) Experimental and numerical simulations of moored self-reacting point absorber wave energy converters. In: Proceedings of the 25 th International Ocean and Polar Engineering Conference, At Kona, Big Island, Hawaii, USA

33. Buckham B, Nahon M, Seto M, Zhao X, Lambert C (2003) Dynamics and control of a towed underwater vehicle system, part I: model development. Ocean Eng 30:453-470

34. Hartog D (2002) Tuned mass damper systems. In: Connor JJ (ed) Introduction to structural motion control, 1st edn. Prentice Hall, New York, pp 217-285

35. Meteorological Observation Stations with Recent Data. https://www. ndbc.noaa.gov/

36. National Oceanic and Atmospheric Administration (NOAA). https://www. noaa.gov/

\section{Publisher's Note}

Springer Nature remains neutral with regard to jurisdictional claims in published maps and institutional affiliations.

\section{Submit your manuscript to a SpringerOpen ${ }^{\circ}$ journal and benefit from:}

- Convenient online submission

- Rigorous peer review

- Open access: articles freely available online

- High visibility within the field

- Retaining the copyright to your article

Submit your next manuscript at $\boldsymbol{\nabla}$ springeropen.com 